“C 2016 IEEE. Personal use of this material is permitted. Permission from IEEE must be obtained for all other uses, in any current or future media, including reprinting/republishing this material for advertising or promotional purposes, creating new collective works, for resale or redistribution to servers or lists, or reuse of any copyrighted component of this work in other works." 


\section{Distributed Dynamic State Estimation Over a Lossy Communication Network with an Application to Smart Grids}

\begin{abstract}
In contrast to the traditional centralised power system state estimation methods, this paper investigates the interconnected optimal filtering problem for distributed dynamic state estimation considering packet losses. Specifically, the power system incorporating microgrids is modelled as a state-space linear equation where sensors are deployed to obtain measurements. Basically, the sensing information is transmitted to the energy management system (EMS) through a lossy communication network where measurements are lost. This can seriously deteriorate the system monitoring performance and even lose network stability. Secondly, as the system states are unavailable, so the estimation is essential to know the overall operating conditions of the electricity network. Availability of the system states provides designers an accurate picture of the power network, so a suitable control strategy can be applied to avoid massive blackouts due to lose network stability. Particularly, the proposed estimator is based on the mean squared error between the actual state and its estimate. To obtain the distributed estimation, the optimal local and neighbouring gains are computed to reach a consensus estimation after exchanging their information with the neighbouring estimators. Then the convergence of the developed algorithm is theoretically proved. Afterwards, a distributed controller is designed based on the semidefinite programming approach. Simulation results demonstrate the accuracy of the developed approaches under the condition of missing measurements.

Index Terms-Distributed controller, distributed dynamic estimation, energy management system, packet losses, linear matrix inequality.
\end{abstract}

\section{INTRODUCTION}

Generally speaking, the industrial domain application becomes more and more distributed due to advanced information and communication technology [1], [2]. In other words, the automation system is mostly designed based on the distributed architecture and its signal processing algorithms. As the measurements are locally processed, it can accurately handle more data, offer flexible communication infrastructure, deliver required functionality and services in sustainable and efficient ways, for example, monitoring and controlling the power system incorporating microgrids in a distributed way. The main reasons of incorporating microgrid including distributed energy resources (DERs) into the grid are due to the low green house gas emissions, reduced transmission losses and cost [3], [4], [5]. Unfortunately, their intermittent nature of the power generation pattern brings critical challenges for power system operation and stability [6].

As the power substations and energy management system (EMS) are generally far away, so the measurements are normally lost in the communication channel [7], [8]. It is therefore imperative to estimate the power system states and apply a suitable control strategy, so the system can operate properly [9]. In other words, the power network intrinsically requires to expect stability over a lossy communication channel between the microgrid and EMS. This can only be archived if the EMS knows the microgrid states as its states are generally unavailable and affected by uncertainties [10]. For instance, in a smart grid the sensing devices such as sensors may be geographically far away from the estimator and controller which inevitable leads to packet dropouts in the communication network. To illustrate, the communication infrastructure with an estimator and a controller at EMS are illustrated in Fig. 1. It can be seen

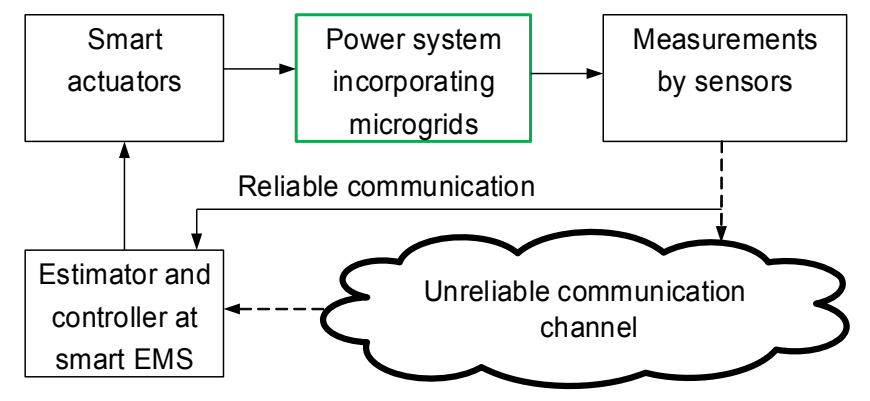

Fig. 1. Regulated power system with communication infrastructure [11].

that the sensing measurements are affected by communication impairments when it transmits through an unreliable communication network [12]. That is, the controller performance and system stability are affected by the impairments [11], [13], [14]. Basically, deregulation of the power network and the advent of smart grid technologies are making EMS feasible and attractive from the customers and utility point of views [15]. These stimulate to present a renewable microgrid model and its distributed estimation with a feedback control strategy under the condition of communication impairments.

\section{A. Related literature}

There is a wealth of research relating to the power system state estimation. To begin with, a distributed weighted least square state estimation method using additive Schwarz domain decomposition technique is proposed in [16]. This decomposition divides the data set into several subsets to reduce the execution time. Unfortunately, it is assumed that the communication is perfect with no measurement losses. A Kalman filter (KF) based state estimation via wireless 
sensor networks over fading channels is presented in [17]. This kind of centralized estimation technique is generally not only in need of huge amount of communication and computation resources but also vulnerable to the central point failures which may lead to massive blackouts. To deal with the communication impairments, a distributed fusion based KF algorithm for sensor networks is developed in [18], [19]. The fusion centre linearly combines the local estimators through a set of designed weighting factors. In order to obtain a suitable weighting factor, a weighted density function based recursive algorithm is purposed under the condition of reliable communication channels [20]. In order to accommodate the effects of random delay in measurements, an extended KF based power system state estimation method is proposed in [21]. All of the aforementioned papers consider the centralised estimation or reliable communication channel.

There are many different feedback control techniques available for the power system stability in the literature. First of all, a linear quadratic Gaussian control strategy under the condition of packet losses is presented in [9]. This networked control system is suitable for the centralized state estimation and its stability analysis. Recently, the time-delay switch attack based on the simple proportional integral derivative (PID) centralized controller is adapted in the context of smart grids [22]. It is considered that delays can be introduced in the sensing loop or control lines so the packet may be lost. Each controller only communicates information with its neighbours in the distributed control strategy [23], [24]. It is therefore very effective for performing the wide-area distributed computation, specifically in the emerging smart grid. Recently, a unified distributed control strategy for the DC microgrid is proposed in [6]. It shows that the standard distributed PI voltage controllers are no longer able to regulate the average DC microgrid bus voltage, so the distributed voltage controllers are replaced by double integrator controllers. Usually, the partial system state information is only available, so the estimation with controller design remain an open question in the signal processing, control and smart grid communities. Authors in [25] developed a novel distributed observer over a lossless channel where the controller is designed at each local area. However, after estimating the system states in a distributed way, the distributed controller generally allows a sparse communication to reduce communication and computation resources while maintaining the system stability. Inspiring by the above discussions and analysis, this paper designs a distributed state estimation and distributed controller under the condition of packet losses.

\section{B. Key contributions}

The main contributions of this research are the following:

- Based on the mean squared error principle, the optimal local and neighbouring gains are determined to obtain a distributed dynamic state estimation in the context of smart grids. Each estimator exchanges information with the neighbouring estimators for reaching a consensus estimation even though there are unmeasurable states and packet losses.
- The convergence of the developed approach is theoretically proved based on the continuous-time domain analysis due to its mathematical simplicity. It shows that the error function is gradually decreased over time, therefore the estimated states converge to the actual states.

- For proper operation and maintaining the stability of the microgrid, a distributed controller is proposed based on the semidefinite programming (SDP) approach. The designed sparse feedback gain is calculated by iterative optimization process which is less conservative as it effectively and efficiently computes the Lyapunov matrix $\mathbf{P}$ with no structure constraints on $\mathbf{P}$.

Notation: Bold face upper and lower case letters are used to represent matrices and vectors respectively. Superscripts $\mathbf{x}^{\prime}$ denotes the transpose of $\mathbf{x}, \operatorname{diag}(\mathbf{x})$ denotes the diagonal matrix, $E(\cdot)$ denotes the expectation operator and $\mathbf{I}$ denotes the identity matrix.

\section{SYSTEM AND PACKET LOSS MODELS}

There is a strong drive in power industry to design, create and analyze the system in distributed ways considering flexible communication infrastructure. In order to develop a distributed estimator and controller, consider the following discrete-time system:

$$
\mathbf{x}_{k+1}=\mathbf{A}_{d} \mathbf{x}_{k}+\mathbf{B}_{d} \mathbf{u}_{k}+\mathbf{n}_{k},
$$

where $\mathbf{x}_{k}$ is the system state at time instant $\mathrm{k}, \mathbf{u}_{k}$ is the control effort and $\mathbf{n}_{k}$ is process noise whose covariance matrix is $\mathbf{Q}_{k}$. The system measurements are obtained by a set of sensors as follows:

$$
\mathbf{z}_{k}^{i}=\mathbf{C}^{i} \mathbf{x}_{k}+\mathbf{w}_{k}^{i},
$$

where $\mathbf{z}_{k}^{i}$ is the observation information by the i-th estimator at time instant $\mathrm{k}$ and $\mathbf{w}_{k}^{i}$ is the measurement noise whose covariance matrix is $\mathbf{R}_{k}^{i}$. Realistically, the sensing measurements transmit through a lossy communication network which causes packet dropouts. This is due to the fact that the power network and EMS are far way from each other. Taking into account the packet loss, (2) can be written as follows:

$$
\mathbf{y}_{k}^{i}=\boldsymbol{\alpha}_{k}^{i} \mathbf{C}^{i} \mathbf{x}_{k}+\boldsymbol{\alpha}_{k}^{i} \mathbf{w}_{k}^{i},
$$

where $\mathbf{y}_{k}^{i}$ is the received measurements under the condition of packet losses, and $\boldsymbol{\alpha}_{k}^{i}=\operatorname{diag}\left[\alpha_{k}^{i}(1), \ldots, \alpha_{k}^{i}\left(r^{i}\right)\right], r^{i}$ is the dimension of $\mathbf{y}_{k}^{i}$ and $\alpha_{k}^{i}(.) \in\{0,1\}$ is the Bernoulli distribution modelled as follows [8]:

$$
\alpha_{k}^{i}(j)= \begin{cases}1, & \text { with probability of } \lambda^{i}(j), \\ 0, & \text { with probability of } 1-\lambda^{i}(j),\end{cases}
$$

where $\lambda^{i}(j)$ is the packet arrival rate reaching at the estimator. For the sake of mathematical simplicity, it is assumed that observation matrices and packet loss distribution are identical each other [26], [27]. Here, we assume $\mathbf{C}^{i}=\mathbf{C}, \alpha_{k}^{i}=\alpha$ and $\lambda^{i}(j)=\lambda(j)$. The assumptions are probable due to the fact that the distributed estimators are not far way from the power substations but as usual information transmits through 
an unreliable network. Secondly, the sensors have limited power and processing capability.

\section{PROPOSED DISTRIBUTED DYNAMIC STATE ESTIMATION ALGORITHM}

Generally speaking, the filtering infrastructure is interconnected to each other to know the operating conditions of the distribution power network. For instance, the proposed interconnected filtering scheme is depicted in Fig. 2. Consid-

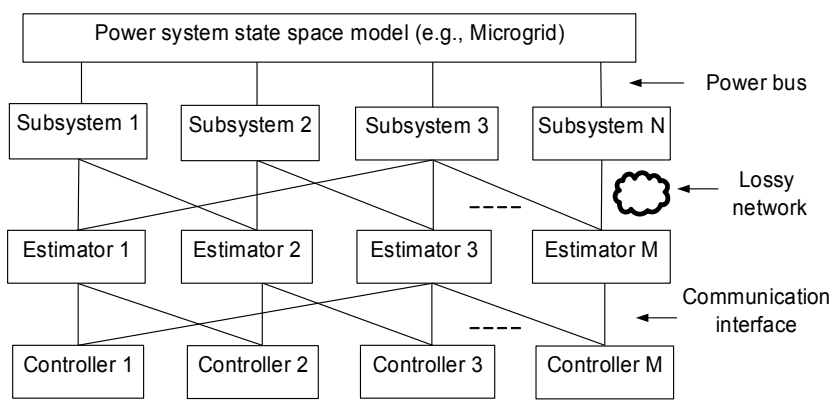

Fig. 2. Proposed interconnected filtering scheme.

ering the packet losses the proposed distributed dynamic state estimator is written as follows:

$$
\begin{aligned}
\hat{\mathbf{x}}_{k \mid k}^{i}=\hat{\mathbf{x}}_{k \mid k-1}^{i}+\mathbf{K}_{k}^{i}\left[\mathbf{y}_{k}^{i}-\right. & \left.\boldsymbol{\alpha} \mathbf{C} \hat{\mathbf{x}}_{k \mid k-1}^{i}\right]+ \\
& \mathbf{L}_{k}^{i} \sum_{j \in N_{k}^{i}}\left[\mathbf{y}_{k}^{j}-\boldsymbol{\alpha} \mathbf{C} \hat{\mathbf{x}}_{k \mid k-1}^{i}\right] .
\end{aligned}
$$

Here, $\hat{\mathbf{x}}_{k \mid k}^{i}$ is the updated state estimation at the i-th estimator, $\hat{\mathbf{x}}_{k \mid k-1}^{i}$ is the predicted states estimate, $\mathbf{K}_{k}^{i}$ is the local gain, $\mathbf{L}_{k}^{i}$ is the neighbouring gain and $N_{k}^{i}$ denotes the set of neighbouring estimators. The second term in (4) is used for local estimation while third term is used to exchange information with neighboring estimators to reach a consensus estimation. Based on the aforementioned modelling structure, our first problem is to design the optimal gains $\mathbf{K}_{k}^{i}$ and $\mathbf{L}_{k}^{i}$, so that the estimated state converges to the actual system state.

Let $\mathbf{e}^{i}$ denotes the estimation error between actual state and estimated state of the i-th estimator, which can be expressed as follows:

$$
\begin{aligned}
& \mathbf{e}_{k \mid k-1}^{i}=\mathbf{x}_{k}-\hat{\mathbf{x}}_{k \mid k-1}^{i}, \\
& \mathbf{e}_{k \mid k}^{i}=\mathbf{x}_{k}-\hat{\mathbf{x}}_{k \mid k}^{i} .
\end{aligned}
$$

Let $n_{k}^{i}=n_{k}^{i}\left(N_{k}^{i}\right)$ represents the cardinality of $N_{k}^{i}$. Now substituting (4) into (6), and using (3) one can obtain the following expression:

$$
\begin{aligned}
\mathbf{e}_{k \mid k}^{i}= & \mathbf{x}_{k}-\hat{\mathbf{x}}_{k \mid k-1}^{i}-\mathbf{K}_{k}^{i}\left[\mathbf{y}_{k}^{i}-\boldsymbol{\alpha} \mathbf{C} \hat{\mathbf{x}}_{k \mid k-1}^{i}\right]- \\
& \mathbf{L}_{k}^{i} \sum_{l \in N_{k}^{i}}\left[\mathbf{y}_{k}^{l}-\boldsymbol{\alpha} \mathbf{C} \hat{\mathbf{x}}_{k \mid k-1}^{i}\right] \\
= & {\left[\mathbf{I}-\boldsymbol{\alpha} \mathbf{K}_{k}^{i} \mathbf{C}-n_{k}^{i} \boldsymbol{\alpha} \mathbf{L}_{k}^{i} \mathbf{C}\right]\left[\mathbf{x}_{k}-\hat{\mathbf{x}}_{k \mid k-1}^{i}\right]-} \\
& \boldsymbol{\alpha} \mathbf{K}_{k}^{i} \mathbf{w}_{k}^{i}-\boldsymbol{\alpha} \mathbf{L}_{k}^{i} \sum_{l \in N_{k}^{i}} \mathbf{w}_{k}^{l} \\
= & {\left[\mathbf{I}-\boldsymbol{\alpha} \mathbf{K}_{k}^{i} \mathbf{C}-n_{k}^{i} \boldsymbol{\alpha} \mathbf{L}_{k}^{i} \mathbf{C}\right] \mathbf{e}_{k \mid k-1}^{i}-\boldsymbol{\alpha} \mathbf{K}_{k}^{i} \mathbf{w}_{k}^{i}-} \\
& \boldsymbol{\alpha} \mathbf{L}_{k}^{i} \sum_{l \in N_{k}^{i}} \mathbf{w}_{k}^{l} .
\end{aligned}
$$

Now the estimation error covariance matrix $\mathbf{P}_{k \mid k}^{i}$ is defined by:

$$
\mathbf{P}_{k \mid k}^{i}=E\left[\mathbf{e}_{k \mid k}^{i} \mathbf{e}_{k \mid k}^{i}\right]
$$

where $E(\cdot)$ is the expectation operator. Substituting (7) into (8), one can obtain:

$$
\begin{aligned}
\mathbf{P}_{k \mid k}^{i} & =\lambda\left[\mathbf{I}-\mathbf{K}_{k}^{i} \mathbf{C}-n_{k}^{i} \mathbf{L}_{k}^{i} \mathbf{C}\right] \mathbf{P}_{k \mid k-1}^{i}\left[\mathbf{I}-\mathbf{K}_{k}^{i} \mathbf{C}-n_{k}^{i} \mathbf{L}_{k}^{i} \mathbf{C}\right]^{\prime} \\
& +(\mathbf{I}-\lambda) \mathbf{P}_{k \mid k-1}^{i}+\lambda \mathbf{K}_{k}^{i} \mathbf{R}_{k}^{i} \mathbf{K}_{k}^{\prime i}+\lambda \mathbf{L}_{k}^{i} \sum_{l \in N_{k}^{i}} \mathbf{R}_{k}^{l} \mathbf{L}_{k}^{\prime}
\end{aligned}
$$

Here, $\mathbf{P}_{k \mid k-1}^{i}=E\left[\mathbf{e}_{k \mid k-1}^{i} \mathbf{e}_{k \mid k-1}^{\prime}\right]$. The following partial derivatives are used to obtain the optimal expression of $\mathbf{K}_{k}^{i}$ and $\mathbf{L}_{k}^{i}$. For any two compatible matrices $\mathbf{X}$ and $\mathbf{Y}$, the following partial derivatives holds:

$$
\begin{aligned}
& \frac{\partial \operatorname{tr}(\mathbf{Y X})}{\partial \mathbf{X}}=\mathbf{Y}^{\prime} \\
& \frac{\partial \operatorname{tr}\left(\mathbf{X Y} \mathbf{X}^{\prime}\right)}{\partial \mathbf{X}}=\mathbf{X}\left(\mathbf{Y}+\mathbf{Y}^{\prime}\right),
\end{aligned}
$$

where $\operatorname{tr}(\cdot)$ is the trace operator. In order to find the optimal gain $\mathbf{K}_{k}^{i}$, taking the partial derivative of $\mathbf{P}_{k \mid k}^{i}$ in (9) with respect to $\mathbf{K}_{k}^{i}$ and applying (10) and (11) yields:

$$
\begin{aligned}
\frac{\partial\left[\operatorname{tr} \mathbf{P}_{k \mid k}^{i}\right]}{\partial \mathbf{K}_{k}^{i}}=- & 2 \lambda\left(\mathbf{I}-n_{k}^{i} \mathbf{L}_{k}^{i} \mathbf{C}\right) \mathbf{P}_{k \mid k-1}^{i} \mathbf{C}^{\prime}+ \\
& 2 \lambda \mathbf{K}_{k}^{i}\left(\mathbf{C P}_{k \mid k-1}^{i} \mathbf{C}^{\prime}+\mathbf{R}_{k}^{i}\right) .
\end{aligned}
$$

Now putting $\frac{\partial\left[\operatorname{tr} \mathbf{P}_{k \mid k}^{i}\right]}{\partial \mathbf{K}_{k}^{i}}=0$ in (12), the optimal local gain $\mathbf{K}_{k}^{i}$ is given by:

$$
\begin{gathered}
\mathbf{K}_{k}^{i}=\left[\mathbf{P}_{k \mid k-1}^{i} \mathbf{C}^{\prime}-n_{k}^{i} \mathbf{L}_{k}^{i} \mathbf{C P}_{k \mid k-1}^{i} \mathbf{C}^{\prime}\right] \\
{\left[\mathbf{C P}_{k \mid k-1}^{i} \mathbf{C}^{\prime}+\mathbf{R}_{k}^{i}\right]^{-1} .}
\end{gathered}
$$

Similarly, take the partial derivative of (9) with respect to $\mathbf{L}_{k}^{i}$ and apply (10) and (11) to obtain:

$$
\begin{aligned}
& \frac{\partial\left[\operatorname{tr} \mathbf{P}_{k \mid k}^{i}\right]}{\partial \mathbf{L}_{k}^{i}}=- 2 n_{k}^{i} \lambda\left(\mathbf{I}-\mathbf{K}_{k}^{i} \mathbf{C}\right)^{\prime} \mathbf{P}_{k \mid k-1}^{i} \mathbf{C}+ \\
& 2\left(n_{k}^{i}\right)^{2} \lambda \mathbf{L}_{k}^{i} \mathbf{C} \mathbf{P}_{k \mid k-1}^{i} \mathbf{C}^{\prime}+2 \lambda \mathbf{L}_{k}^{i} \sum_{l \in N_{k}^{i}} \mathbf{R}_{k}^{l} .
\end{aligned}
$$


Setting $\frac{\partial\left[t r \mathbf{P}_{k \mid k}^{i}\right]}{\partial \mathbf{L}_{k}^{i}}=0$ in (14), $\mathbf{L}_{k}^{i}$ is derived as follows:

$$
\begin{gathered}
\mathbf{L}_{k}^{i}=\left[n_{k}^{i} \mathbf{P}_{k \mid k-1}^{i} \mathbf{C}^{\prime}-n_{k}^{i} \mathbf{K}_{k}^{i} \mathbf{C} \mathbf{P}_{k \mid k-1}^{i} \mathbf{C}^{\prime}\right] \\
{\left[\left(n_{k}^{i}\right)^{2} \mathbf{C P}_{k \mid k-1}^{i} \mathbf{C}^{\prime}+\sum_{l \in N_{k}^{i}} \mathbf{R}_{k}^{l}\right]^{-1} .}
\end{gathered}
$$

For simplicity, define $\mathbf{H}_{k}^{i}=\mathbf{C} \mathbf{P}_{k \mid k-1}^{i} \mathbf{C}^{\prime}, \mathbf{F}_{k}^{i}=\left(\mathbf{H}_{k}^{i}+\mathbf{R}_{k}^{i}\right)^{-1}$, and $\mathbf{G}_{k}^{i}=\left[\left(n_{k}^{i}\right)^{2} \mathbf{H}_{k}^{i}+\sum_{l \in N_{k}^{i}} \mathbf{R}_{k}^{l}\right]^{-1}$. Then (13) and (15) can be rewritten as follows:

$$
\begin{aligned}
\mathbf{K}_{k}^{i} & =\left[\mathbf{P}_{k \mid k-1}^{i} \mathbf{C}^{\prime}-n_{k}^{i} \mathbf{L}_{k}^{i} \mathbf{C} \mathbf{P}_{k \mid k-1}^{i} \mathbf{C}^{\prime}\right] \mathbf{F}_{k}^{i} \\
& =\mathbf{P}_{k \mid k-1}^{i} \mathbf{C}^{\prime} \mathbf{F}_{k}^{i}-n_{k}^{i} \mathbf{L}_{k}^{i} \mathbf{H}_{k}^{i} \mathbf{F}_{k}^{i} . \\
\mathbf{L}_{k}^{i} & =\left[n_{k}^{i} \mathbf{P}_{k \mid k-1}^{i} \mathbf{C}^{\prime}-n_{k}^{i} \mathbf{K}_{k}^{i} \mathbf{C P}_{k \mid k-1}^{i} \mathbf{C}^{\prime}\right] \mathbf{G}_{k}^{i} \\
& =n_{k}^{i} \mathbf{P}_{k \mid k-1}^{i} \mathbf{C}^{\prime} \mathbf{G}_{k}^{i}-n_{k}^{i} \mathbf{K}_{k}^{i} \mathbf{H}_{k}^{i} \mathbf{G}_{k}^{i} .
\end{aligned}
$$

In order to obtain the optimal gain $\mathbf{K}_{k}^{i}$, substituting (17) into (16) leads to:

$$
\begin{aligned}
& \mathbf{K}_{k}^{i}= \mathbf{P}_{k \mid k-1}^{i} \mathbf{C}^{\prime} \mathbf{F}_{k}^{i}-n_{k}^{i}\left[n_{k}^{i} \mathbf{P}_{k \mid k-1}^{i} \mathbf{C}^{\prime} \mathbf{G}_{k}^{i}-n_{k}^{i} \mathbf{K}_{k}^{i} \mathbf{H}_{k}^{i} \mathbf{G}_{k}^{i}\right] \mathbf{H}_{k}^{i} \mathbf{F}_{k}^{i} \\
&= \mathbf{P}_{k \mid k-1}^{i} \mathbf{C}^{\prime} \mathbf{F}_{k}^{i}-\left(n_{k}^{i}\right)^{2} \mathbf{P}_{k \mid k-1}^{i} \mathbf{C}^{\prime} \mathbf{G}_{k}^{i} \mathbf{H}_{k}^{i} \mathbf{F}_{k}^{i} \\
& \quad+\left(n_{k}^{i}\right)^{2} \mathbf{K}_{k}^{i} \mathbf{H}_{k}^{i} \mathbf{G}_{k}^{i} \mathbf{H}_{k}^{i} \mathbf{F}_{k}^{i} \\
& \Rightarrow \mathbf{K}_{k}^{i}=\left[\begin{array}{c}
\left.\mathbf{P}_{k \mid k-1}^{i} \mathbf{C}^{\prime} \mathbf{F}_{k}^{i}-\left(n_{k}^{i}\right)^{2} \mathbf{P}_{k \mid k-1}^{i} \mathbf{C}^{\prime} \mathbf{G}_{k}^{i} \mathbf{H}_{k}^{i} \mathbf{F}_{k}^{i}\right] \\
{\left[\mathbf{I}-\left(n_{k}^{i}\right)^{2} \mathbf{H}_{k}^{i} \mathbf{G}_{k}^{i} \mathbf{H}_{k}^{i} \mathbf{F}_{k}^{i}\right]^{-1} .}
\end{array}\right.
\end{aligned}
$$

Similarly,

$$
\begin{gathered}
\mathbf{L}_{k}^{i}=\left[n_{k}^{i} \mathbf{P}_{k \mid k-1}^{i} \mathbf{C}^{\prime} \mathbf{G}_{k}^{i}-n_{k}^{i} \mathbf{P}_{k \mid k-1}^{i} \mathbf{C}^{\prime} \mathbf{F}_{k}^{i} \mathbf{H}_{k}^{i} \mathbf{G}_{k}^{i}\right] \\
{\left[\mathbf{I}-\left(n_{k}^{i}\right)^{2} \mathbf{H}_{k}^{i} \mathbf{F}_{k}^{i} \mathbf{H}_{k}^{i} \mathbf{G}_{k}^{i}\right]^{-1} .}
\end{gathered}
$$

In summary, after initialization the system parameters such as $\mathbf{P}_{k \mid k-1}^{i}$ and $\hat{\mathbf{x}}_{k \mid k-1}^{i}$ through KF based prediction step, each estimator computes the optimal local and neighbouring gains by (18) and (19). $\hat{\mathbf{x}}_{k+1 \mid k}^{i}$ and $\mathbf{P}_{k+1 \mid k}^{i}$ are given by:

$$
\begin{gathered}
\hat{\mathbf{x}}_{k+1 \mid k}^{i}=\mathbf{A}_{d} \hat{\mathbf{x}}_{k \mid k}^{i}+\mathbf{B}_{d} u_{k}, \\
\mathbf{P}_{k+1 \mid k}^{i}=\mathbf{A}_{d} \mathbf{P}_{k \mid k}^{i} \mathbf{A}_{d}^{\prime}+\mathbf{Q}_{k} .
\end{gathered}
$$

Afterwards, each estimator computes the state estimation and its update covariance matrix by (4) and (9). Now our next problem is to guarantee the consensus of the proposed distributed state estimation method, so that the developed approach can also apply to other real-time applications.

\section{Consensus analysis}

From the engineering perspective, the discrete-time system is easy to implement in the digital platforms, while the continuous system is easy to analyze from the mathematical point of view [28]. Motivated by this realistic dilemma and similar to [29], the consensus analysis of the proposed algorithm is completed based on the consensus analysis of the continuous system. Similar to the discrete-time case, the estimator applies the following step:

$$
\dot{\hat{\mathbf{x}}}^{i}=\mathbf{A} \hat{\mathbf{x}}^{i}+\mathbf{B u}+\mathbf{K}^{i}\left[\mathbf{y}^{i}-\boldsymbol{\alpha} \mathbf{C} \hat{\mathbf{x}}^{i}\right]+\mathbf{L}^{i} \sum_{j \in N^{i}}\left[\mathbf{y}^{j}-\boldsymbol{\alpha} \mathbf{C} \hat{\mathbf{x}}^{i}\right] .
$$

The estimation error $\mathbf{e}^{i}$ can be expressed as follows:

$$
\mathbf{e}^{i}=\mathbf{x}-\hat{\mathbf{x}}^{i} .
$$

By direct differentiation of (23), the estimation error dynamics is in the following form:

$$
\begin{aligned}
\mathbf{e}^{i}=\dot{\mathbf{x}}-\dot{\mathbf{x}}^{i} & \\
= & \dot{\mathbf{x}}-\mathbf{A} \hat{\mathbf{x}}^{i}-\mathbf{B u}-\mathbf{K}^{i}\left[\mathbf{y}^{i}-\boldsymbol{\alpha} \mathbf{C} \hat{\mathbf{x}}^{i}\right]-\mathbf{L}^{i} \sum_{j \in N^{i}}\left[\mathbf{y}^{j}-\boldsymbol{\alpha} \mathbf{C} \hat{\mathbf{x}}^{i}\right] \\
= & \mathbf{A x}+\mathbf{B u}+\mathbf{n}-\mathbf{A} \hat{\mathbf{x}}^{i}-\mathbf{B u}-\boldsymbol{\alpha} \mathbf{K}^{i}\left[\mathbf{C} \mathbf{x}+\mathbf{w}^{i}-\mathbf{C} \hat{\mathbf{x}}^{i}\right]- \\
& \quad \mathbf{L}^{i} \sum_{j \in N^{i}}\left[\mathbf{y}^{j}-\boldsymbol{\alpha} \mathbf{C} \hat{\mathbf{x}}^{i}\right] \\
= & \left(\mathbf{A}-\boldsymbol{\alpha} \mathbf{K}^{i} \mathbf{C}-n^{i} \boldsymbol{\alpha} \mathbf{L}^{i} \mathbf{C}\right) \mathbf{x}-\left(\mathbf{A}-\boldsymbol{\alpha} \mathbf{K}^{i} \mathbf{C}-n^{i} \boldsymbol{\alpha} \mathbf{L}^{i} \mathbf{C}\right) \hat{\mathbf{x}}^{i}+ \\
& \quad \mathbf{n}-\boldsymbol{\alpha} \mathbf{K}^{i} \mathbf{w}^{i}-\boldsymbol{\alpha} \mathbf{L}^{i} \sum_{l \in N^{i}} \mathbf{w}^{l} \\
= & \left(\mathbf{A}-\boldsymbol{\alpha} \mathbf{K}^{i} \mathbf{C}-n^{i} \boldsymbol{\alpha} \mathbf{L}^{i} \mathbf{C}\right)\left(\mathbf{x}-\hat{\mathbf{x}}^{i}\right)+\mathbf{n}-\boldsymbol{\alpha} \mathbf{K}^{i} \mathbf{w}^{i}-\boldsymbol{\alpha} \mathbf{L}^{i} \sum_{l \in N^{i}} \mathbf{w}^{l} \\
= & \left(\mathbf{A}-\boldsymbol{\alpha} \mathbf{K}^{i} \mathbf{C}^{i}-n^{i} \boldsymbol{\alpha} \mathbf{L}^{i} \mathbf{C}\right) \mathbf{e}^{i}+\mathbf{n}-\boldsymbol{\alpha}^{i} \mathbf{K}^{i} \mathbf{w}^{i}-\boldsymbol{\alpha}^{i} \mathbf{L}^{i} \sum_{l \in N^{i}} \mathbf{w}^{l} .
\end{aligned}
$$

The error covariance matrix is written as follows:

$$
\begin{aligned}
& \dot{\mathbf{P}^{i}}=\left(\mathbf{A}-\lambda \mathbf{K}^{i} \mathbf{C}-n^{i} \lambda \mathbf{L}^{i} \mathbf{C}\right) \mathbf{P}^{i}+\mathbf{P}^{i}\left(\mathbf{A}-\lambda \mathbf{K}^{i} \mathbf{C}-n^{i} \lambda \mathbf{L}^{i} \mathbf{C}\right)^{\prime}+ \\
& \mathbf{Q}+\lambda \mathbf{K}^{i} \mathbf{R}^{i} \mathbf{K}^{\prime i}+\lambda \mathbf{L}^{i} \sum_{l \in N^{i}} \mathbf{R}^{l} \mathbf{L}^{\prime i} \\
& =\mathbf{A} \mathbf{P}^{i}+\mathbf{P}^{i} \mathbf{A}^{\prime}+\mathbf{Q}-\lambda \mathbf{K}^{i} \mathbf{C} \mathbf{P}^{i}-\lambda \mathbf{P}^{i} \mathbf{C}^{\prime} \mathbf{K}^{\prime i}+\lambda \mathbf{K}^{i} \mathbf{R}^{i} \mathbf{K}^{\prime i} \\
& -n^{i} \lambda \mathbf{L}^{i} \mathbf{C} \mathbf{P}^{i}-n^{i} \lambda \mathbf{P}^{i} \mathbf{C}^{\prime} \mathbf{L}^{i}+\lambda \mathbf{L}^{i} \sum_{l \in N^{i}} \mathbf{R}^{l} \mathbf{L}^{i} .
\end{aligned}
$$

Taking the partial derivative of (25) with respect to $\mathbf{K}^{i}$ and applying (10) and (11) yields:

$$
\frac{\partial\left[\operatorname{tr} \dot{\mathbf{P}}^{i}\right]}{\partial \mathbf{K}^{i}}=-2 \lambda \mathbf{P}^{i} \mathbf{C}^{\prime}+2 \lambda \mathbf{K}^{i} \mathbf{R}^{i}
$$

Setting $\frac{\partial\left[t r \dot{\mathbf{P}}^{i}\right]}{\partial \mathbf{K}^{i}}=0$ in (26), then the gain matrix is given by:

$$
\mathbf{K}^{i}=\mathbf{P}^{i} \mathbf{C}^{\prime}\left(\mathbf{R}^{i}\right)^{-1} .
$$

Taking the partial derivative of (25) with respect to $\mathbf{L}^{i}$ and applying (10) and (11) leads to:

$$
\frac{\partial\left[t r \dot{\mathbf{P}}^{i}\right]}{\partial \mathbf{L}^{i}}=-2 n^{i} \lambda \mathbf{P}^{i} \mathbf{C}^{\prime}+2 \lambda \mathbf{L}^{i} \sum_{l \in N^{i}} \mathbf{R}^{l} .
$$

Putting $\frac{\partial\left[t \dot{\mathbf{P}}^{i}\right]}{\partial \mathbf{L}^{i}}=0$ in (28), then the gain matrix is obtained as follows:

$$
\mathbf{L}^{i}=n^{i} \mathbf{P}^{i} \mathbf{C}^{\prime}\left(\sum_{l \in N^{i}} \mathbf{R}^{l}\right)^{-1}
$$


Substituting (27) and (29) into (25), one can obtain:

$$
\begin{aligned}
\dot{\mathbf{P}^{i}=} & \mathbf{A} \mathbf{P}^{i}+\mathbf{P}^{i} \mathbf{A}^{\prime}+\mathbf{Q}-\lambda \mathbf{P}^{i} \mathbf{C}^{\prime}\left(\mathbf{R}^{i}\right)^{-1} \mathbf{C} \mathbf{P}^{i}- \\
& \lambda \mathbf{P}^{i} \mathbf{C}^{\prime}\left(\mathbf{R}^{i}\right)^{-1} \mathbf{C} \mathbf{P}^{i}+\lambda \mathbf{P}^{i} \mathbf{C}^{\prime}\left(\mathbf{R}^{i}\right)^{-1} \mathbf{C P}^{i}- \\
& n^{i} \lambda \mathbf{P}^{i} \mathbf{C}^{\prime}\left(\sum_{l \in N^{i}} \mathbf{R}^{l}\right)^{-1} \mathbf{C P}^{i}-n^{i} \lambda \mathbf{P}^{i} \mathbf{C}^{\prime}\left(\sum_{l \in N^{i}} \mathbf{R}^{l}\right)^{-1} \mathbf{C} \mathbf{P}^{i}+ \\
& \left(n^{i}\right)^{2} \lambda \mathbf{P}^{i} \mathbf{C}^{\prime}\left(\sum_{l \in N^{i}} \mathbf{R}^{l}\right)^{-1} \mathbf{C} \mathbf{P}^{i} \\
= & \mathbf{A} \mathbf{P}^{i}+\mathbf{P}^{i} \mathbf{A}^{\prime}+\mathbf{Q}-\lambda \mathbf{P}^{i} \mathbf{C}^{\prime}\left(\mathbf{R}^{i}\right)^{-1} \mathbf{C P}^{i}+ \\
& {\left[\left(n^{i}\right)^{2}-2 n^{i}\right] \lambda \mathbf{P}^{i} \mathbf{C}^{\prime}\left(\sum_{l \in N^{i}} \mathbf{R}^{l}\right)^{-1} \mathbf{C P}^{i} }
\end{aligned}
$$

In order to analyze the stability of the developed approach, define $\overline{\mathbf{e}}^{i}=E\left(\mathbf{e}^{i}\right)$, and take the expectation on both sides of (24) to obtain:

$$
\dot{\overline{\mathbf{e}}}^{i}=\left(\mathbf{A}-\lambda^{i} \mathbf{K}^{i} \mathbf{C}^{i}-n^{i} \lambda \mathbf{L}^{i} \mathbf{C}\right) \overline{\mathbf{e}}^{i} .
$$

Consider the following Lyapunov function:

$$
V=\sum_{i=1}^{M} \overline{\mathbf{e}}^{i}\left(\mathbf{P}^{i}\right)^{-1} \overline{\mathbf{e}}^{i} .
$$

Now taking the partial derivative and expectation of (32), and using (27), (29), (30) and (31), we have:

$$
\begin{aligned}
& \dot{V}=\sum_{i=1}^{M}\left\{\dot{\overline{\mathbf{e}}}^{i}\left(\mathbf{P}^{i}\right)^{-1} \overline{\mathbf{e}}^{i}+\overline{\mathbf{e}}^{i}\left(\mathbf{P}^{i}\right)^{-1} \dot{\overline{\mathbf{e}}}^{i}-\overline{\mathbf{e}}^{i}\left(\mathbf{P}^{i}\right)^{-1} \dot{\mathbf{P}}^{i}\left(\mathbf{P}^{i}\right)^{-1} \overline{\mathbf{e}}^{i}\right\} \\
& =\sum_{i=1}^{M} \overline{\mathbf{e}}^{\prime} i\left[\left(\mathbf{A}-\lambda \mathbf{K}^{i} \mathbf{C}-n^{i} \lambda \mathbf{L}^{i} \mathbf{C}\right)^{\prime}\left(\mathbf{P}^{i}\right)^{-1}+\left(\mathbf{P}^{i}\right)^{-1}\left(\mathbf{A}-\lambda \mathbf{K}^{i} \mathbf{C}\right.\right. \\
& \left.-n^{i} \lambda \mathbf{L}^{i} \mathbf{C}\right)-\left(\mathbf{P}^{i}\right)^{-1} \mathbf{A}-\mathbf{A}^{\prime}\left(\mathbf{P}^{i}\right)^{-1}-\left(\mathbf{P}^{i}\right)^{-1} \mathbf{Q}\left(\mathbf{P}^{i}\right)^{-1} \\
& \left.+\lambda \mathbf{C}^{\prime}\left(\mathbf{R}^{i}\right)^{-1} \mathbf{C}-\left[\left(n^{i}\right)^{2}-2 n^{i}\right] \lambda \mathbf{C}^{\prime}\left(\sum_{l \in N^{i}} \mathbf{R}^{l}\right)^{-1} \mathbf{C}\right] \overline{\mathbf{e}}^{i} \\
& =\sum_{i=1}^{M} \overline{\mathbf{e}}^{\prime i}\left[-\lambda\left(\mathbf{P}^{i}\right)^{-1} \mathbf{K}^{i} \mathbf{C}-\lambda \mathbf{C}^{\prime} \mathbf{K}^{\prime i}\left(\mathbf{P}^{i}\right)^{-1}-\right. \\
& n^{i} \lambda\left(\mathbf{P}^{i}\right)^{-1} \mathbf{L}^{i} \mathbf{C}-n^{i} \lambda \mathbf{C}^{\prime} \mathbf{L}^{\prime i}\left(\mathbf{P}^{i}\right)^{-1}- \\
& \left(\mathbf{P}^{i}\right)^{-1} \mathbf{Q}\left(\mathbf{P}^{i}\right)^{-1}+\lambda \mathbf{C}^{\prime}\left(\mathbf{R}^{i}\right)^{-1} \mathbf{C} \\
& \left.-\left[\left(n^{i}\right)^{2}-2 n^{i}\right] \lambda \mathbf{C}^{\prime}\left(\sum_{l \in N^{i}} \mathbf{R}^{l}\right)^{-1} \mathbf{C}\right] \overline{\mathbf{e}}^{i} \\
& =\sum_{i=1}^{M} \overline{\mathbf{e}}^{\prime} i\left[-\lambda \mathbf{C}^{\prime}\left(\mathbf{R}^{i}\right)^{-1} \mathbf{C}-\lambda \mathbf{C}^{\prime}\left(\mathbf{R}^{i}\right)^{-1} \mathbf{C}-\right. \\
& n^{i} \lambda \mathbf{C}^{\prime}\left(\sum_{l \in N^{i}} \mathbf{R}^{l}\right)^{-1} \mathbf{C}-n^{i} \lambda \mathbf{C}^{\prime}\left(\sum_{l \in N^{i}} \mathbf{R}^{l}\right)^{-1} \mathbf{C}- \\
& \left(\mathbf{P}^{i}\right)^{-1} \mathbf{Q}\left(\mathbf{P}^{i}\right)^{-1}+\lambda \mathbf{C}^{\prime}\left(\mathbf{R}^{i}\right)^{-1} \mathbf{C} \\
& \left.-\left[\left(n^{i}\right)^{2}-2 n^{i}\right] \lambda \mathbf{C}^{\prime}\left(\sum_{l \in N^{i}} \mathbf{R}^{l}\right)^{-1} \mathbf{C}\right] \overline{\mathbf{e}}^{i} \\
& =-\sum_{i=1}^{M} \overline{\mathbf{e}}^{\prime i}\left[\lambda \mathbf{C}^{\prime}\left(\mathbf{R}^{i}\right)^{-1} \mathbf{C}+\left(\mathbf{P}^{i}\right)^{-1} \mathbf{Q}\left(\mathbf{P}^{i}\right)^{-1}\right. \\
& \left.+\left(n^{i}\right)^{2} \lambda \mathbf{C}^{\prime}\left(\sum_{l \in N^{i}} \mathbf{R}^{l}\right)^{-1} \mathbf{C}\right] \overline{\mathbf{e}}^{i}<0 .
\end{aligned}
$$

Consequently, the estimated state $\hat{\mathbf{x}}^{i}$ converges to the actual system state $\mathbf{x}$. After estimating the system states, the designer needs to apply a suitable distributed control technique for maintaining the stability of the network.

\section{Proposed Distributed CONTROL STRATEgy}

Generally, computing machines have finite memory and temporal resolution [22], so the distributed controller is obviously preferred from the engineering aspects. The feedback controller is employed to regulate the microgrid states, which is given by:

$$
\mathbf{u}_{k}=\mathbf{F} \hat{\mathbf{x}}_{k \mid k} .
$$

Here, $\mathbf{F}$ is the distributed feedback gain matrix to be designed. If there is no connection between subsystem/estimator and controller then the corresponding element of $\mathbf{F}$ is zero. For instance, from the Fig. 2 the designed gain matrix $\mathbf{F}$ belongs to the following structure set (assuming ${ }^{1} \mathrm{M}=4$ ):

$$
\mathbf{F}^{\circ}=\left\{\mathbf{F} \mid \mathbf{F}=\left[\begin{array}{cccc}
F_{11} & F_{12} & 0 & 0 \\
0 & F_{22} & F_{23} & 0 \\
F_{31} & 0 & F_{33} & F_{34} \\
0 & 0 & 0 & F_{44}
\end{array}\right]\right\} .
$$

Here, the feedback element $F_{N M}$ is the connection between subsystem sensor $\mathrm{N}$ and controller $\mathrm{M}$. This type of distributed feedback gain structure offers sparse communication between the grid and EMS [6]. Consequently, sensors can share information with their neighbouring controllers only, which reduces communication overheads and offers flexible controlling infrastructure over centralised control strategies.

According to the separation principle [30, p. 427], we can implement the control law $\mathbf{u}_{k}=\mathbf{F} \mathbf{x}_{k}$ [9]. Using $\mathbf{u}_{k}$, (1) can be written as follows:

$$
\mathbf{x}_{k+1}=\tilde{\mathbf{A}}_{d} \mathbf{x}_{k}+\mathbf{n}_{k},
$$

where $\tilde{\mathbf{A}}_{d}=\mathbf{A}_{d}+\mathbf{B}_{d} \mathbf{F}$ is the closed loop state matrix. If there exists a stablizing gain matrix $\mathbf{F} \in \mathbf{F}^{\circ}$, then the following LMI holds:

$$
\begin{aligned}
& \tilde{\mathbf{A}}_{d}^{\prime} \mathbf{P} \tilde{\mathbf{A}}_{d}-\mathbf{P}<\mathbf{0} \\
& \left(\mathbf{A}_{d}+\mathbf{B}_{d} \mathbf{F}\right)^{\prime} \mathbf{P}\left(\mathbf{A}_{d}+\mathbf{B}_{d} \mathbf{F}\right)-\mathbf{P}<\mathbf{0} .
\end{aligned}
$$

In order to obtain a feasible solution so that the distributed feedback can be applied, $\mathbf{P}$ is computed as follows:

$$
\left(\beta \mathbf{A}_{d}\right)^{\prime} \mathbf{P}\left(\beta \mathbf{A}_{d}\right)-\mathbf{P}<\mathbf{0},
$$

where $\beta=1 /\left[\gamma \max \left\{\operatorname{eig}\left(\mathbf{A}_{d}\right)\right\}\right], \gamma>1$ is a free parameter and $\max \left\{\operatorname{eig}\left(\mathbf{A}_{d}\right)\right\}$ is the maximum eigen values of $\mathbf{A}_{d}$. The quantity $\gamma$ ensures eigenvalues of the scaled close loop system strictly less than one. Now according to the standard Schur's complement, (38) can be transformed into the following LMI form:

$$
\left[\begin{array}{cc}
-\mathbf{P} & \beta \mathbf{A}_{d}^{\prime} \mathbf{P} \\
\beta \mathbf{P} \mathbf{A}_{d} & -\mathbf{P}
\end{array}\right]<\mathbf{0} .
$$

\footnotetext{
${ }^{1}$ The proposed work can be easily extended to the generic case.
} 
After computing $\mathbf{P}$ in (39) and with the help of (37), one can obtain $\mathbf{F} \in \mathbf{F}^{\circ}$ by considering the following optimization problem:

$$
\begin{array}{ll}
\text { minimise } & \zeta \\
\text { subject to } & \left(\mathbf{A}_{d}+\mathbf{B}_{d} \mathbf{F}\right)^{\prime} \mathbf{P}\left(\mathbf{A}_{d}+\mathbf{B}_{d} \mathbf{F}\right)-\mathbf{P}+\zeta \mathbf{I}<\mathbf{0 .}
\end{array}
$$

where $\zeta$ is the semidefinite programming variable. Given $\mathbf{P}$, applying the Schur's complement to (40) yields:

$$
\left[\begin{array}{cc}
-\mathbf{P}+\zeta \mathbf{I} & \left(\mathbf{A}_{d}+\mathbf{B}_{d} \mathbf{F}\right)^{\prime} \mathbf{P} \\
\mathbf{P}\left(\mathbf{A}_{d}+\mathbf{B}_{d} \mathbf{F}\right) & -\mathbf{P}
\end{array}\right]<\mathbf{0} .
$$

Finally, one can formulate the proposed optimization problem as follows:

$$
\begin{array}{ll}
\text { minimise } & \zeta \\
\text { subject to } & H o l d \\
\text { (41), } \mathbf{F} \in \mathbf{F}^{\circ} .
\end{array}
$$

In summary, the proposed feedback gain is designed by solving (42). As the feedback structure offers sparse communication, the developed approach reduces computation costs.

\section{APPLICATION TO THE DISTRIBUTION POWER SYSTEM AND SIMULATION RESULTS}

In this section, the proposed algorithm is applied to the microgrid state estimation and control in the context of smart grids.

\section{A. Distribution power system incorporating microgrid}

Due to the climate change and limited energy resources, the renewable microgrid incorporating DERs is integrated into the main grid at the point common couplings (PCC). As their power generation pattern are generally intermittent in nature, so it needs to monitor the PCC voltages and keep it at a reference value by applying a suitable control technique [31]. Generally speaking, the microgrid may also be install in the remote and mountain areas, so its monitoring and stability should be managed in a distributed way. Driven by these motivations, the considered $\mathrm{N}$ micro-sources are connected to the main grid. It is assumed that $\mathrm{N}=4$ solar panels are connected through the IEEE-4 bus distribution system shown in Fig. 3 [31], [32]. Here, the input voltages are defined by

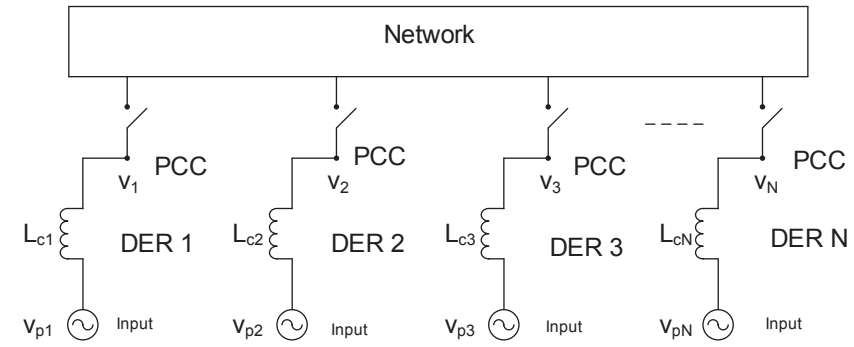

Fig. 3. Solar panels are connected to the distribution power network [31].

$\mathbf{v}_{p}=\left(\begin{array}{llll}v_{p 1} & v_{p 2} & v_{p 3} & v_{p 4}\end{array}\right)^{\prime}$, where $v_{p i}$ is the i-th DER input voltage. It can be seen that the considered four micro-sources are connected to the power network at the corresponding PCCs whose voltages are defined by $\mathbf{v}_{s}=\left(\begin{array}{llll}v_{1} & v_{2} & v_{3} & v_{4}\end{array}\right)^{\prime}$, where $v_{i}$ is the i-th PCC voltage. Note that the system in Fig. 3 is not restrictive, and can be more general cases; the methods proposed in this paper is independent of the type and size of microgrids.

The nodal voltage equation is obtained by applying the Laplace transformations as follows:

$$
\mathbf{Y}(s) \mathbf{v}_{s}(s)=\frac{1}{s} \mathbf{L}_{c}^{-1} \mathbf{v}_{p}(s),
$$

where $\mathbf{L}_{c}=\operatorname{diag}\left(L_{c_{1}}, L_{c_{2}}, L_{c_{3}}, L_{c_{4}}\right)$ and $\mathbf{Y}(s)$ is the admittance matrix of the entire power network incorporating four mico-sources. Based on the standard specifications of the IEEE 4-bus system [31], the admittance matrix is given in (44). Now we can convert the transfer function into the following form [31]:

$$
\dot{\mathbf{x}}_{t}=\mathbf{A} \mathbf{x}_{t}+\mathbf{B} \mathbf{u}_{t}+\mathbf{n}_{t}
$$

Here, the system state $\mathbf{x}_{t}=\mathbf{v}_{s}-\mathbf{v}_{\text {ref }}$ is the PCC voltage deviation, $\mathbf{v}_{\text {ref }}$ is the PCC reference voltage, $\mathbf{u}_{t}=\mathbf{v}_{p}-\mathbf{v}_{\text {pref }}$ is the DER control effort deviation, $\mathbf{v}_{\text {pref }}$ is the reference control effort, $\mathbf{n}_{t}$ is the process noise whose covariance matrix is $\mathbf{Q}_{t}$, the system state matrix $\mathbf{A}$, and input matrix $\mathbf{B}$ are given by:

$$
\begin{aligned}
\mathbf{A} & =\left[\begin{array}{cccc}
175.9 & 176.8 & 511 & 103.6 \\
-350 & 0 & 0 & 0 \\
-544.2 & -474.8 & -408.8 & -828.8 \\
-119.7 & -554.6 & -968.8 & -1077.5
\end{array}\right], \\
\mathbf{B} & =\left[\begin{array}{cccc}
0.8 & 334.2 & 525.1 & -103.6 \\
-350 & 0 & 0 & 0 \\
-69.3 & -66.1 & -420.1 & -828.8 \\
-434.9 & -414.2 & -108.7 & -1077.5
\end{array}\right] .
\end{aligned}
$$

Now the system model (45) is expressed as a discrete-time state-space linear equation (1) where system state matrix $\mathbf{A}_{d}=\mathbf{I}+\mathbf{A} \Delta t, \mathbf{I}$ is the identity matrix, $\Delta t$ is the sampling period and $\mathbf{B}_{d}=\mathbf{B} \Delta t$ and $\mathbf{n}_{d}=\mathbf{n}_{t} \Delta t$ with covariance matrix $\mathbf{Q}=\mathbf{Q}_{t} \Delta t^{2}$. Based on the aforementioned algorithm and power network, the simulation is carried out for validity of the theoretical analysis.

\section{B. Numerical results}

The simulation is conducted through Matlab and YALMIP and the parameters are shown in Table I.

TABLE I

THE PARAMETERS FOR THE SIMULATION USING MATLAB.

\begin{tabular}{cccc}
\hline Parameters & Values & Parameters & Values \\
\hline $\mathbf{Q}$ & $0.0000001 * \mathbf{I}_{4}$ & $\mathbf{R}^{1}$ & $0.000001 * \mathbf{I}_{4}$ \\
$\mathbf{R}^{2}$ & $0.000002 * \mathbf{I}_{4}$ & $\mathbf{R}^{3}$ & $0.000003 * \mathbf{I}_{4}$ \\
$\mathbf{R}^{4}$ & $0.000004 * \mathbf{I}_{4}$ & $\lambda$ & 0.95 \\
$\gamma$ & 2 & $\Delta t$ & 0.0001 \\
\hline
\end{tabular}

From the simulation, the system state versus time step results are demonstrated in Figs. 4-7. It can be observed that the packet loss significantly affects the system states but the proposed algorithm can well estimate the system states. 
$\mathbf{Y}(s)=\left(\mathbf{L}_{c} s\right)^{-1}+\left[\begin{array}{cccc}\frac{1}{0.1750+0.0005 s} & \frac{-1}{0.1750+0.0005 s} & 0 & 0 \\ \overline{0.1750+0.0005 s} & \frac{1}{0.1750+0.0005 s}+\frac{-1}{0.1667+0.0004 s} & \frac{-1}{0.1667+0.0004 s} & 0 \\ 0 & \frac{-1}{0.1667+0.0004 s} & \frac{1}{0.1667+0.0004 s}+\frac{-1}{0.2187+0.0006 s} & \frac{1}{0.2187+0.0006 s} \\ 0 & 0 & \overline{0.2187+0.0006 s} & \frac{1}{0.2187+0.0006 s}+\frac{1}{12.3413+0.0148 s} \\ (44)\end{array}\right]$.

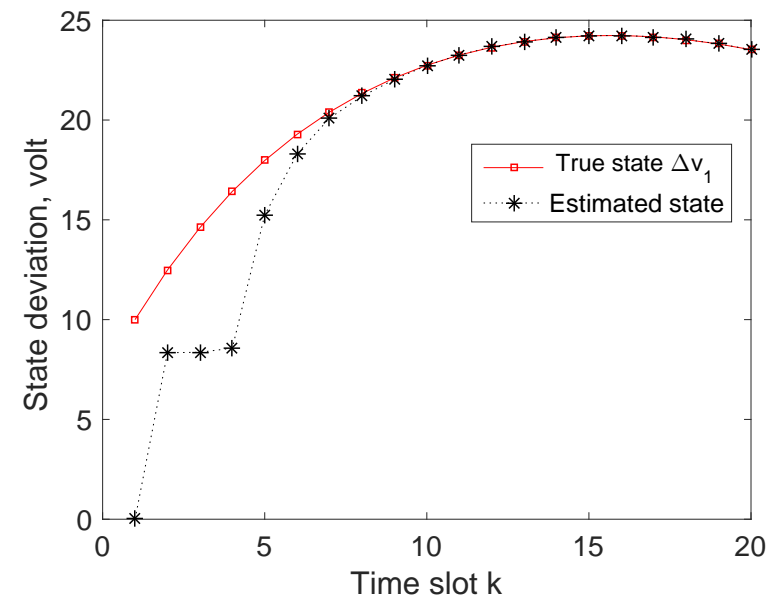

Fig. 4. State trajectory of $\Delta v_{1}$ and its estimate.

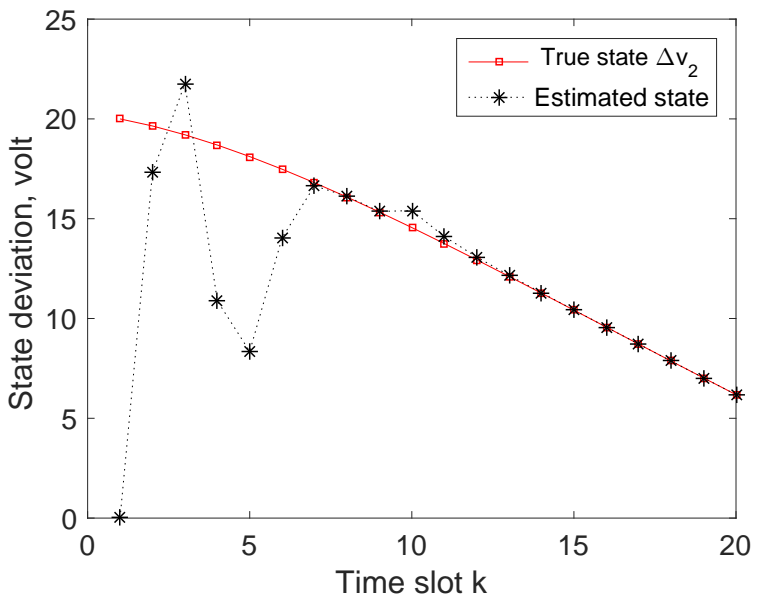

Fig. 5. State trajectory of $\Delta v_{2}$ and its estimate.

This is due to the fact that the proposed algorithm can find the optimal gains to extract the system state information from adversaries. It can also be seen that it requires only 0.015 seconds $(k \times \Delta t)$ to estimate the system states which are much less than the standard estimation time frame of 1 second [33]. Note that the small fluctuation comes from the packet losses and random noises.

Basically, it can be seen that the actual PCC state deviations increase dramatically over time, which is very dangerous in terms of power network stability and microgrid operation.

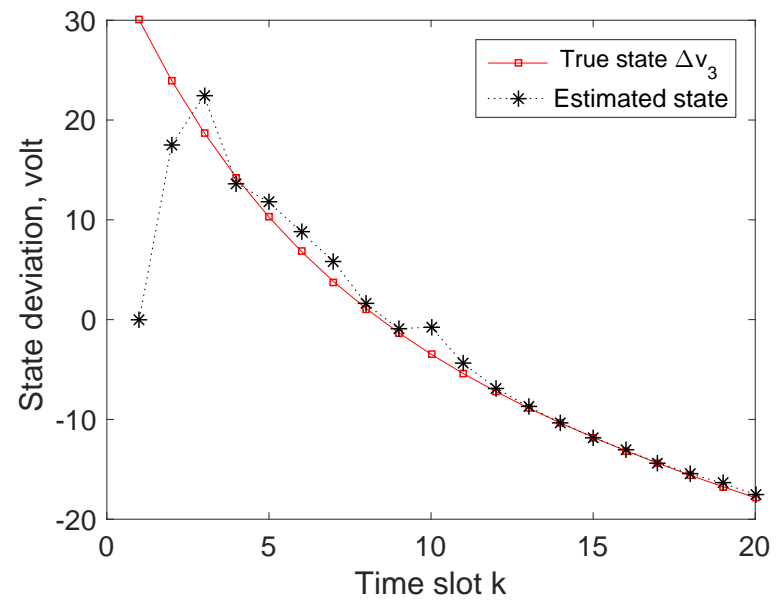

Fig. 6. State trajectory of $\Delta v_{3}$ and its estimate.

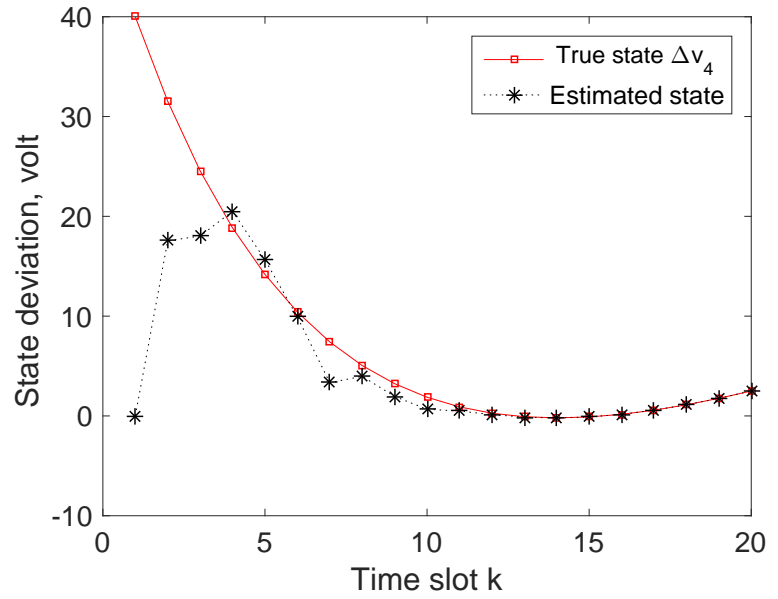

Fig. 7. State trajectory of $\Delta v_{4}$ and its estimate.

Thus, it is intrinsically essential to apply a suitable control technique, so that the PCC voltage deviations are driven to zero in a fairly short time. After applying the proposed distributed control method, it can be seen from Fig. 8 that the proposed controller is able to keep the voltage deviations to zero in approximately 0.01 seconds $(k \times \Delta t)$, which acts as a precursor for stability and microgrid operations. Technically, it means that the developed approach requires much less time to keep the voltage as a reference value compared with the standard stability time frame $1-5$ seconds [33]. 


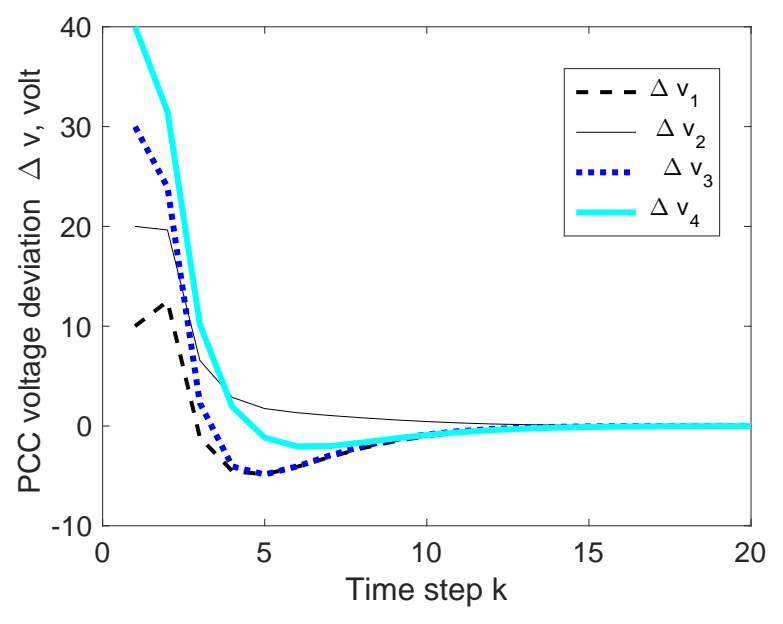

Fig. 8. Controlling the states trajectory.

\section{CONCLUSION}

This paper presents a distributed state estimation and control method considering packet losses. The developed distributed consensus estimator is based on the mean squared error, so it can accurately compute the optimal gains to extract the actual system states. Finally, in order to regulate the system states, this study proposes a semidefinite programming based distributed controller in the context of smart grid communication. The proposed distributed control framework could properly determine the sparse gain such that the system states will be stabilized in a fairly short time. These approaches can help to design the future smart EMS under the condition of uncertainties. It is worth pointing out that the aforementioned problems are not trivial in the smart grid community as the communication impairments have significant impact in the grid stability and the distributed strategies can reduce communication burdens and offer sparse communication network.

\section{REFERENCES}

[1] R. Vadigepalli III and F. J. Doyle, "A distributed state estimation and control algorithm for plantwide processes," IEEE Transactions on Control Systems Technology, vol. 11, no. 1, pp. 119-127, 2003.

[2] J. Chen, X. Cao, P. Cheng, Y. Xiao, and Y. Sun, "Distributed collaborative control for industrial automation with wireless sensor and actuator networks," IEEE Transactions on Industrial Electronics, vol. 57, no. 12, pp. 4219-4230, 2010.

[3] E. R. Diaz, J. C. V. Quintero, and J. M. Guerrero, "Intelligent DC homes in future sustainable energy systems: When efficiency and intelligence work together," IEEE Consumer Electronics Magazine, vol. 5, no. 1, pp. 74-80, 2016.

[4] C. Wan, J. Zhao, Y. Song, Z. Xu, J. Lin, and Z. Hu, "Photovoltaic and solar power forecasting for smart grid energy management," CSEE Journal of Power and Energy Systems, vol. 1, no. 4, pp. 38-46, 2015.

[5] T. Strasser, F. Andrén, J. Kathan, C. Cecati, C. Buccella, P. Siano, P. Leitao, G. Zhabelova, V. Vyatkin, P. Vrba et al., "A review of architectures and concepts for intelligence in future electric energy systems," IEEE Transactions on Industrial Electronics, vol. 62, no. 4, pp. 2424-2438, 2015.

[6] T. Morstyn, B. Hredzak, G. D. Demetriades, and V. G. Agelidis, "Unified distributed control for DC microgrid operating modes," IEEE Transactions on Power Systems, vol. 31, no. 1, pp. 802-812, 2016.
[7] S. Deshmukh, B. Natarajan, and A. Pahwa, "State estimation over a lossy network in spatially distributed cyber-physical systems," IEEE Transactions on Signal Processing, vol. 62, no. 15, pp. 3911-3923, 2014.

[8] B. Sinopoli, L. Schenato, M. Franceschetti, K. Poolla, M. Jordan, and S. Sastry, "Kalman filtering with intermittent observations," IEEE Transactions on Automatic Control, vol. 49, no. 9, pp. 1453-1464, 2004.

[9] A. K. Singh, R. Singh, and B. C. Pal, "Stability analysis of networked control in smart grids," IEEE Transactions on Smart Grid, vol. 6, no. 1, pp. 381-390, 2015.

[10] E. Ghahremani and I. Kamwa, "Online state estimation of a synchronous generator using unscented Kalman filter from phasor measurements units," IEEE Transactions on Energy Conversion, vol. 26, no. 4, pp. 1099-1108, 2011.

[11] X. Zhang, C. Lu, X. Xie, and Z. Y. Dong, "Stability analysis and controller design of a wide-area time-delay system based on the expectation model method," IEEE Transactions on Smart Grid, vol. 7, no. 1, pp. $520-529,2016$

[12] L. Schenato, "Optimal estimation in networked control systems subject to random delay and packet drop," IEEE Transactions on Automatic Control, vol. 53, no. 5, pp. 1311-1317, 2008.

[13] S. Wen, X. Yu, Z. Zeng, and J. Wang, "Event-triggering load frequency control for multi-area power systems with communication delays," IEEE Transactions on Industrial Electronics, vol. 63, no. 2, pp. 1308-1317, 2016.

[14] W. Yao, L. Jiang, J. Wen, Q. Wu, and S. Cheng, "Wide-area damping controller for power system interarea oscillations: A networked predictive control approach," IEEE Transactions on Control Systems Technology, vol. 23, no. 1, pp. 27-36, 2015.

[15] M. C. Bozchalui, C. A. Cañizares, and K. Bhattacharya, "Optimal operation of climate control systems of produce storage facilities in smart grids," IEEE Transactions on Smart Grid, vol. 6, no. 1, pp. 351$359,2015$.

[16] H. Karimipour and V. Dinavahi, "Parallel domain decomposition based distributed state estimation for large-scale power systems," in Proc. of the Industrial and Commercial Power Systems Technical Conference, 2015, pp. 1-5.

[17] D. E. Quevedo and A. Ahlen, "A predictive power control scheme for energy efficient state estimation via wireless sensor networks," in Proc. of the Decision and Control, 2008, pp. 1103-1108.

[18] B. Chen, W.-A. Zhang, and L. Yu, "Distributed fusion estimation with missing measurements, random transmission delays and packet dropouts," IEEE Transactions on Automatic Control, vol. 59, no. 7, pp. 1961-1967, 2014.

[19] M. Moayedi, Y. K. Foo, and Y. C. Soh, "Adaptive Kalman filtering in networked systems with random sensor delays, multiple packet dropouts and missing measurements," IEEE Transactions on Signal Processing, vol. 58, no. 3, pp. 1577-1588, 2010.

[20] Z.-g. Yin, C. Zhao, Y.-R. Zhong, and J. Liu, "Research on robust performance of speed-sensorless vector control for the induction motor using an interfacing multiple-model extended kalman filter," IEEE Transactions on Power Electronics, vol. 29, no. 6, pp. 3011-3019, 2014.

[21] C.-L. Su and C.-N. Lu, "Interconnected network state estimation using randomly delayed measurements," IEEE Transactions on Power Systems, vol. 16, no. 4, pp. 870-878, 2001.

[22] A. Sargolzaei, K. K. Yen, and M. N. Abdelghani, "Preventing time-delay switch attack on load frequency control in distributed power systems," IEEE Transactions on Smart Grid, vol. 7, no. 2, pp. 1176-1185, 2016.

[23] M. Tahir and S. K. Mazumder, "Self-triggered communication enabled control of distributed generation in microgrids," IEEE Transactions on Industrial Informatics, vol. 11, no. 2, pp. 441-449, 2015.

[24] H. Li and Z. Han, "Distributed scheduling of wireless communications for voltage control in micro smart grid," in Proc. of the GLOBECOM Workshops, 2011, pp. 1188-1193.

[25] T. N. Pham, H. Trinh, and L. V. Hien, "Load frequency control of power systems with electric vehicles and diverse transmission links using distributed functional observers," IEEE Transactions on Smart Grid, vol. 7, no. 1, pp. 238-252, 2016.

[26] R. Ambrosino, B. Sinopoli, K. Poolla, and S. Sastry, "Optimal sensor density for remote estimation over wireless sensor networks," in Proc. of the International Annual Allerton Conference, 2008, pp. 599-606.

[27] R. Olfati-Saber, "Distributed Kalman filter with embedded consensus filters," in Proc. of the International Conference on Decision and Control, 2005, pp. 8179-8184. 
[28] E. Aranda-Escolastico, M. Guinaldo, and S. Dormido, "Stability of output event-based control systems through quadratic trigger functions," in Proc. of the International Conference on Emerging Technologies and Factory Automation, 2015, pp. 1-7.

[29] R. Olfati-Saber, "Distributed Kalman filtering for sensor networks," in Proc. of the Conference on Decision and Control, 2007, pp. 5492-5498.

[30] M. Gopal, Digital Control and State Variable methods Conventional and Neural-Fuzzy Control System. New Delhi, India: McGraw-Hill, 2003.

[31] H. Li, L. Lai, and H. V. Poor, "Multicast routing for decentralized control of cyber physical systems with an application in smart grid," IEEE Journal on Selected Areas in Communications, vol. 30, no. 6, pp. 1097-1107, 2012.

[32] H. Li, F. Li, Y. Xu, D. T. Rizy, and J. D. Kueck, "Adaptive voltage control with distributed energy resources: Algorithm, theoretical analysis, simulation, and field test verification," IEEE Transactions on Power Systems, vol. 25, no. 3, pp. 1638-1647, 2010.

[33] Y. Wang, P. Yemula, and A. Bose, "Decentralized communication and control systems for power system operation," IEEE Transactions on Smart Grid, vol. 6, no. 2, pp. 885-893, 2015. 